\title{
Development of elbow rehabilitation equipment using pneumatic muscles
}

\author{
Georgiana Vetrice $^{1, *}$, and Andrea Deaconescu ${ }^{1}$ \\ ${ }^{1}$ Transilvania University of Brașov, Department of Industrial Engineering and Management, 5 Mihai \\ Viteazul Street, Brașov, Romania
}

\begin{abstract}
Applying continuous passive movements (CPM) to the injured joints represents a viable solution for the rehabilitation of patients with posttraumatic disabilities of the elbow. In order to effectively implement CPM-based rehabilitation procedures, the development of new solutions for high performance equipment serves the interest of both medical facilities and patients. This paper deals with a study of the kinematics, construction and actuation of such new rehabilitation equipment using continuous passive motion. The actuation of this equipment is achieved by two pairs of linear pneumatic muscles.
\end{abstract}

\section{Introduction}

The elbow is one of the most regularly used joints in the body [1]. It is a synovial hinge joint, formed by the lower part of the humerus and the upper part of the radius and ulna. Situated between the shoulder and the hand joint, the mobility of this joint is subordinated to the function of the shoulder and contributes to the mobilization of the hand. Its functional importance derives from the fact that after having sustained elbow injuries, regardless of the cause, both daily activities and professional tasks are compromised.

\section{Biomechanics of the elbow}

Designing such rehabilitation equipment requires knowledge about the anatomy and biomechanics of the upper limb, and especially of the elbow.

The elbow joint possesses two degrees of freedom. It is responsible for the movements known as flexion-extension and pronation-supination [2]. As shown in Fig. 1, flexion consists in rotating the forearm towards the arm, by a maximum angle of $150^{\circ}$. The functional range of motion for elbow is, however, of $30^{\circ}$ to $130^{\circ}$. Extension consists in rotating the arm in the opposite direction, by a maximum angle of $10^{\circ}$ (hyperextension). Fig. 2 shows the pronation-supination movement, which consists in rotating the forearm by its longitudinal axis, by a maximum angle of $90^{\circ}$; the thumb is moving closer to the body in the case of pronation and is turned away from the body in the case of supination. The functional motion for elbow pronation-supination ranges from $50^{\circ}$ to $-50^{\circ}$.

\footnotetext{
* Corresponding author: vetrice.georgiana@unitbv.ro
} 


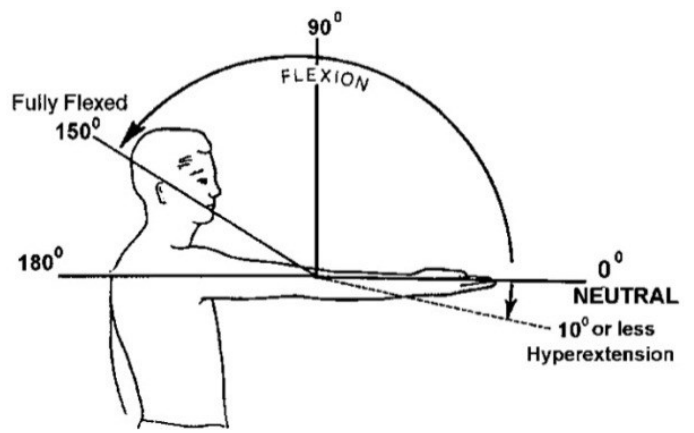

Fig. 1. Elbow flexion-extension [3].
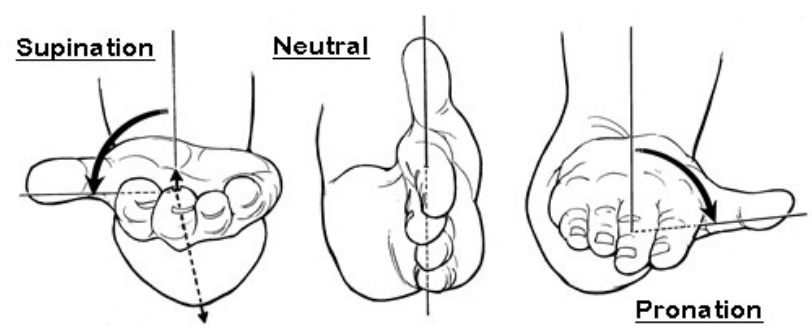

Fig. 2. Elbow pronation-supination [4].

Diminution or loss of elbow functions may be caused by a variety of diseases and medical conditions. Some of these are tendinitis, bursitis, fractures, dislocation, arthritis, etc. Posttraumatic pathologies are the most severe. The goal of posttraumatic rehabilitation is to achieve an optimum level of independent functioning for the patient. The main objective of posttraumatic recovery is to maintain the normal range of motion of the healthy segments, to rehabilitate the parts injured by trauma and to regain the original functions of joint.

The most important method used in posttraumatic recovery is physical therapy. Continuous passive motion, as part of the so-called passive physical therapy, is designed for patients suffering from posttraumatic affections of the elbow who cannot move autonomously. This method consists in applying constant motion to the joint without requiring any effort from the patient.

\section{Design of the elbow rehabilitation equipment}

The elbow movements that need to be recovered are exactly the movements that the rehabilitation equipment should provide. Although rehabilitation equipment is nowadays available on the market, it is driven by electric motors entailing less than favourable dynamic characteristics.

Pneumatic artificial muscles represent an alternative driving modality of these devices. Compared to the most frequently encountered drives at present, their price is lower, they benefit from a better weight-to-power ratio, are adjustable during the rehabilitation process and due to their specific properties the interaction with patients is safe [5].

Among the advantages of using such equipment is the faster recovery of the patients, safe and easy use, increased patient comfort, better rehabilitation results and the possibility of deployment in both medical units and in patients' homes.

Performing a kinematic analysis of the elbow is very important for designing the rehabilitation equipment, because the kinematics of the rehabilitation equipment must 
reflect the kinematics of the joint it is intended for. Fig. 3 shows the modified kinematic model of the elbow.
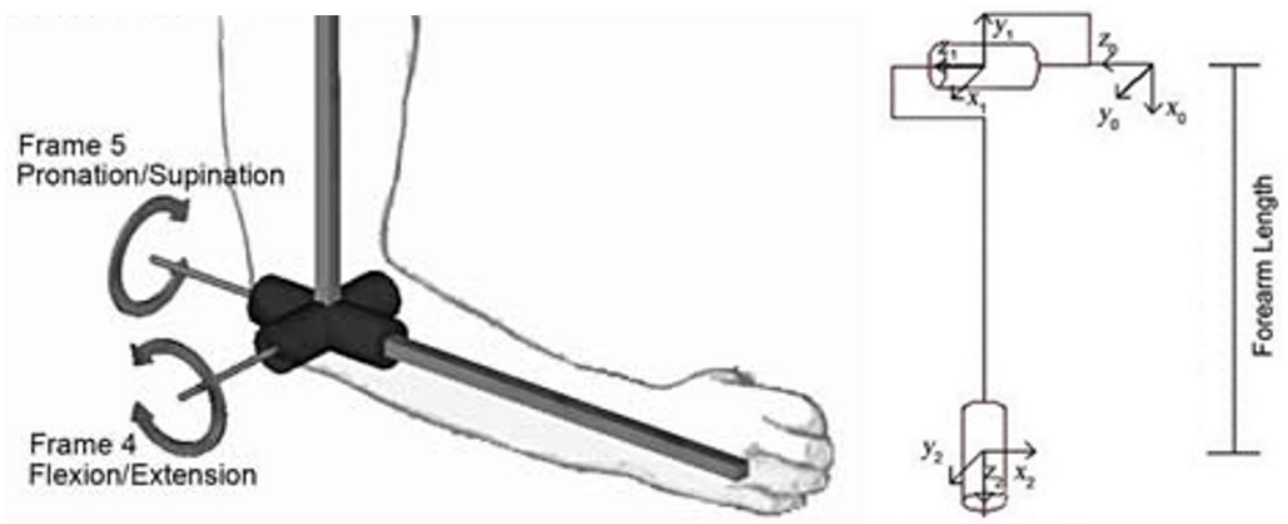

Fig. 3. Modified kinematic model of the elbow [6].

The rehabilitation equipment must have the following characteristics [7]:

- $\quad$ kinematics that allow the replication of human motions.

- $\quad$ ergonomic design, for a comfortable human-machine interaction.

- $\quad$ safety and compliance.

Taking into account the requirements of the rehabilitation process and the kinematic analysis of the elbow, a variant of elbow rehabilitation equipment was designed, the novelty of which consists in the utilization of linear pneumatic muscles and in allowing movement in two planes (Fig. 4).

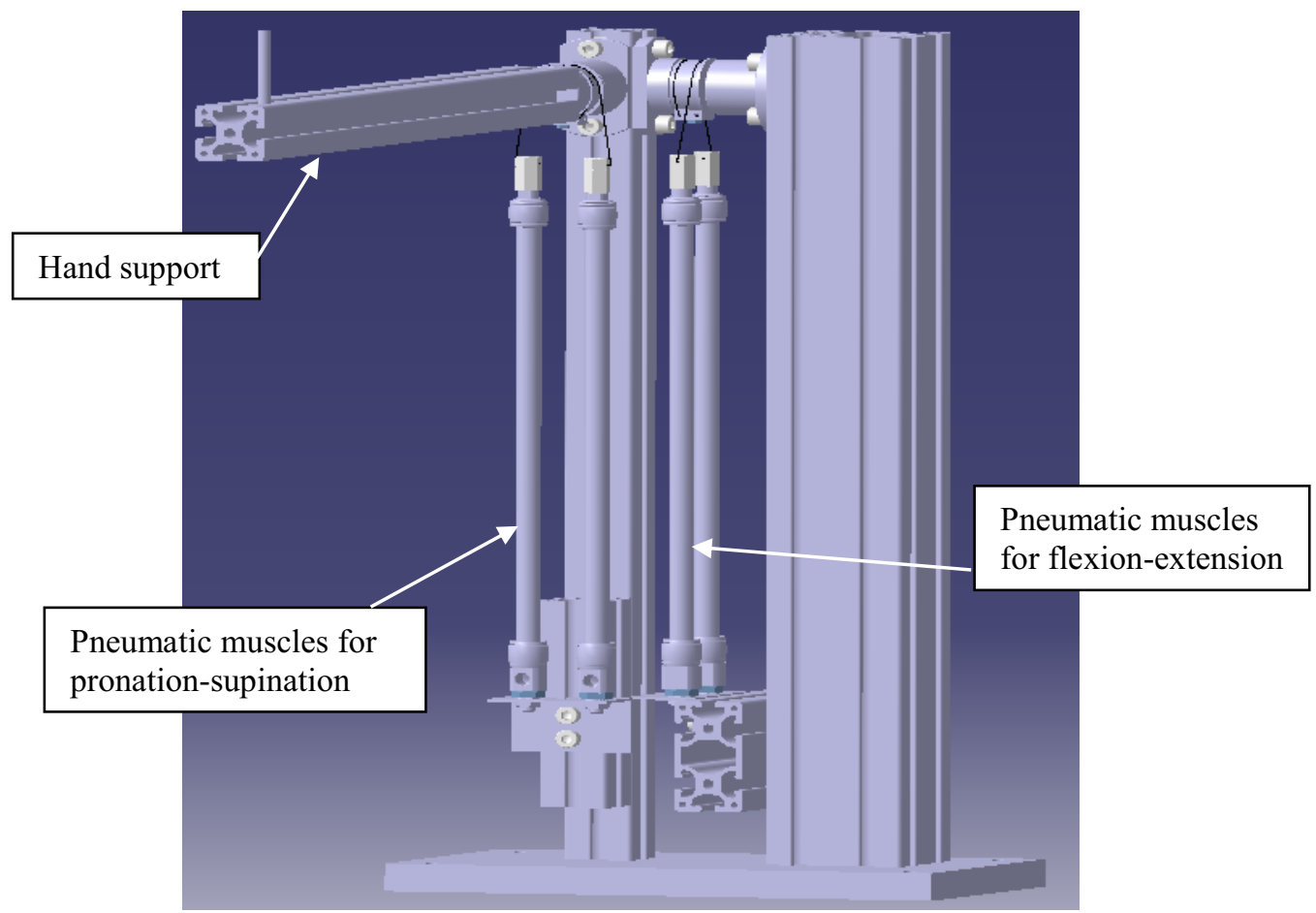

Fig. 4. Elbow rehabilitation equipment. 
As stated above, the linkage of the equipment has to be compatible with linkage of the human limb. The axes of the joint of the equipment have to be identical to the human joint axes, in order to ensure an ergonomic human-machine interaction. During the rehabilitation process, the equipment has to ensure maximum comfort to the patient and must not compromise or limit for any reason the patient's ability to perform natural motions.

Fig. 5 shows the construction of the rotation modules for the rehabilitation equipment. The hand support is adjustable in length for different sizes of the upper limb. Patients place their forearm on the hand support and start the rehabilitation exercise by holding the bar. The upper ends of the pneumatic muscles are connected by a steel cable, drawn over a pulley and fixed on it. These cables ensure the transmission of motion between actuators and joints. The pneumatic muscles work one against the other, causing the rotation of the shaft and also the movement of the hand support. It is important to mention that the movements performed by the equipment can be achieved only separately and not simultaneously.

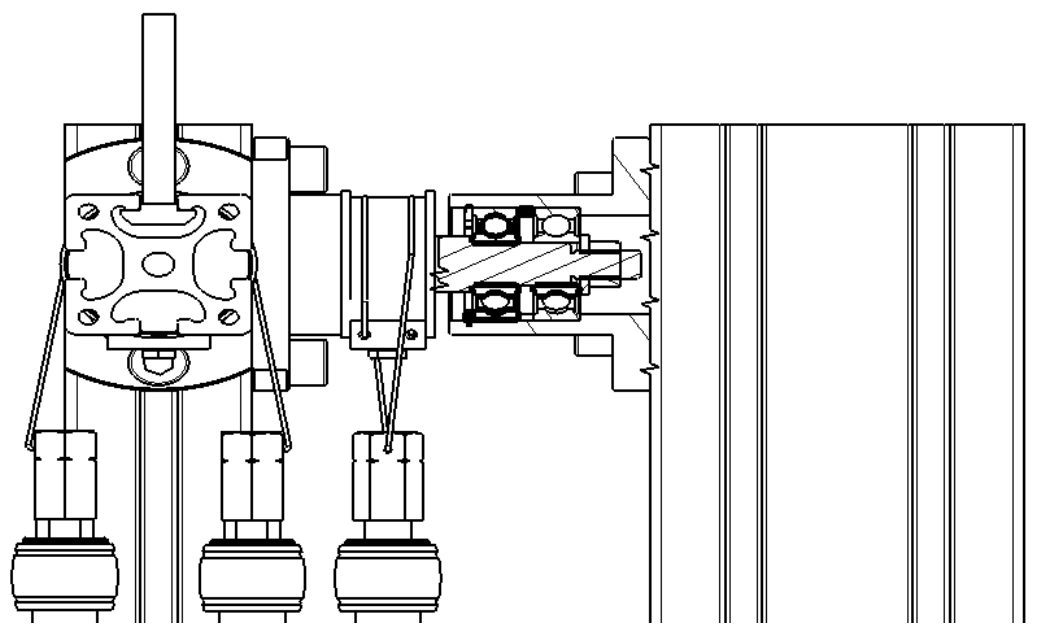

Fig. 5. Breakout view of the rotation module of the rehabilitation equipment.
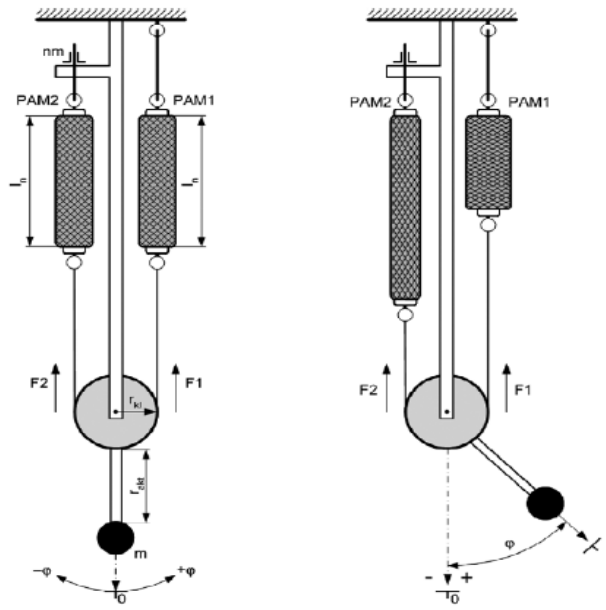

Fig. 6. Pneumatic artificial muscles in antagonistic connection [9].

The equipment has two rotational degrees of freedom, placed perpendicularly. In this case two pairs of muscles were used, one for each movement performed by the equipment. 
Thus, each rotation module of the equipment is driven by a couple of pneumatic muscles arranged in antagonistic connection, as shown in Fig. 6. For achieving the neutral position both muscles are fed the same pressure, and the feeding of compressed air causes the movement to take place.

The four pneumatic muscles used in the construction of the equipment are of $10 \mathrm{~mm}$ interior diameter and $300 \mathrm{~mm}$ initial length. The maximum possible stroke that can be performed is of approximately $20 \%$ of the relaxed muscle length, in this case $60 \mathrm{~mm}$.

\section{Conclusions}

The elbow is a joint that easily develops stiffness, slowing down the rehabilitation process. Regaining the mobility of this joint is indispensable for the mobility of the upper limb; else, a non-rehabilitated elbow can have a major impact on personal and professional life. This desideratum underlies the need for conceiving high performance and cost effective rehabilitation equipment for patients suffering from posttraumatic affections of the elbow joint. The ones developed so far are electrically driven and have a rigid structure.

The research discussed in this paper is aimed at developing a mono-articular device based on continuous passive motion, with two degrees of freedom, which ensures a complete recovery (flexion-extension and pronation-supination, one at a time, not simultaneously). This equipment has also the advantage of being deployable in both medical units and in patients' homes.

This paper presents and discusses the kinematics, construction and actuation system for such rehabilitation equipment, intended for patients lacking the necessary biological resources for achieving the movement. Its design combines knowledge from anatomy, biomechanics and kinematics. The specific requirements for this equipment are due to the fact that it comes into direct contact with patients and also due to its non-conventional drive - pneumatic muscles. Two pairs of linear pneumatic muscles were used in this case, one for each movement. This type of drive is suitable for medical recovery due to its low weight, high performance and low cost.

Currently the rehabilitation equipment is undergoing a test phase, in order to verify the compliant behaviour of these actuators.

\section{References}

1. ***http://www.healthline.com/human-body-maps/elbow\#seoBlock, accessed: 11.02 .2017

2. B.F. Morrey, J. Sanchez-Sotelo, The elbow and its disorders (The Mayo Clinic Foundation, 2009)

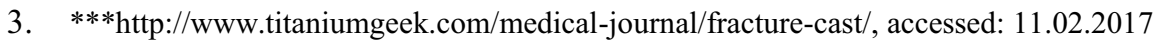

4. $\quad * * *$ https://tt.tennis-warehouse.com/index.php?threads/pronation-and-isr-clarification-questionfor-chas_tennis.546324/, accessed: 11.02.2017

5. B. Jobbagy, D. Simsik, J. Marek, J. Karchnak, D. Onofrejova, AJME, 7, 299 (2014)

6. H.A. Abdullah, C. Tarry, R. Datta, G.S. Mittal, M. Abderrahim, JRRD, 44, 43 (2007)

7. O. Liska, M. More, D. Janacova, H. Charvatova, MMOTE, 151 (2013)

8. T. Deaconescu, A. Deaconescu, Applications of pneumatic muscles developed within the Festo National Fluid Actuation and Automation Training Centre in Brașov (available at http://www.recentonline.ro/050/Deaconescu-R50.pdf, 2016)

9. J. Pitel, M. Balara, AT\&P, 4 (2009) 